\title{
Respiratory muscle dysfunction and exercise limitation in patients with moderate adolescent idiopathic scoliosis
}

\author{
E Marco $^{1 *}$, JM Martínez-Llorens ${ }^{2}$, SC Chiarella ${ }^{3}$, MF Donaire $^{3}$, M Orozco-Levi $^{4}$, F Escalada $^{3}$ \\ From 8th International Conference on Conservative Management of Spinal Deformities and SOSORT 2011 \\ Annual Meeting \\ Barcelona, Spain. 19-21 May 2011
}

\section{Background}

Adolescent idiophatic scoliosis (AIS) can lead to ventilatory restriction, respiratory muscle weakness and exercise limitation. The aim of our study is to describe muscle weakness in AIS patients and its correlation with the curve magnitude.

\section{Materials and methods}

Case-control study in with 85 patients with AIS and 25 healthy volunteers. AIS patients were classified into two groups determined by the curve mangitude): A (Cobb angle $\left.25-40^{\circ}\right)$ and $\mathrm{B}\left(\mathrm{Cobb}\right.$ angle $\left.>40^{\circ}\right)$. Main outcomes were: respiratory muscle strength estimated by maximal inspiratory and expiratory preassures (MIP, MEP), peripherical muscle strength assessed in hands and lower limb muscles, respiratory function tests and exercise capacity. Statistical analysis: chi square test, t-Student and Pearson correlation coefficient.

\section{Results}

The skeletal muscle function was decreased in AIS patients in comparison with the controls $(\mathrm{p}<0.001)$ : MIP (\%) 69.4 (SD 5.12) in Group A and 71 (SD 19) in Group $\mathrm{B}$; in the control group MIP was 95 (SD 15); MEP (\%) 63.2 (SD 17.9) and 69 (SD 19) for A and B respectively. In the control group was 91 (SD 18). We also decreased strength in lower limbs compared with the controls. There appeared to be no connection between spinal deformity and muscle function.

\section{Conclusions}

The patients with AIS show a generalised muscle dysfunction, which contributes to the reduction in their exercise capacity, in absence of a correlation with the magnitude of spinal deformity.

\section{Acknowledgments \\ Partially funded by grants of Fondo de Investigación Sanitaria (PI 070194), SEPAR, OCAP and CIBERES.}

\section{Author details}

'Physical Medicine and Rehabilitation Dpt. Hospitales Mar-Esperança, Parc de Salut Mar. 2. Research Group, Injury, Immune Response and Pulmonary Function. IMIM Barcelona, Spain. ${ }^{2}$ Pneumology Dpt. Hospital del Mar. Parc de Salut Mar; CIBERES (CIBER Respiratory Diseases, ISCIII). Barcelona, Spain. ${ }^{3}$ Physical Medicine and Rehabilitation Dpt. Hospitals Mar-Esperança, Parc de Salut Mar. Barcelona, Spain. ${ }^{4}$ Research Group, Injury, Immune Response and Pulmonary Function. IMIM.; Pneumology Dpt. Hospital del Mar. Parc de Salut Mar; CIBERES (CIBER Respiratory Diseases, ISCIII). Barcelona, Spain.

Published: 27 January 2012

\section{doi:10.1186/1748-7161-7-S1-062}

Cite this article as: Marco et al:: Respiratory muscle dysfunction and exercise limitation in patients with moderate adolescent idiopathic scoliosis. Scoliosis 2012 7(Suppl 1):062.

'Physical Medicine and Rehabilitation Dpt. Hospitales Mar-Esperança, Parc de Salut Mar. 2. Research Group, Injury, Immune Response and Pulmonary Function. IMIM Barcelona, Spain

Full list of author information is available at the end of the article 